\title{
Feature Dimensionality Reduction using a Dual Level Metaheuristic Algorithm
}

\author{
Babatunde R. S. \\ Department of Computer, \\ Library and Information \\ Science. \\ Kwara State University, \\ Malete. Nigeria
}

\author{
Olabiyisi S.O. \\ Department of Computer \\ Science and Engineering. \\ Ladoke Akintola University of \\ Technology, Ogbomoso, \\ Nigeria.
}

\author{
Ganiyu R. A \\ Department of Computer \\ Science and Engineering, \\ Ladoke Akintola University of \\ Technology, Ogbomoso, \\ Nigeria.
}

\author{
Omidiora E.O. \\ Department of Computer \\ Science and Engineering \\ Ladoke Akintola University of \\ Technology, Ogbomoso, \\ Nigeria.
}

\section{All Authors are correspondent Authors}

\begin{abstract}
The need to reduce the curse of dimensionality has spurred active research in the field of pattern recognition and machine learning. High dimensional data culminates in redundant as well as discriminant features. A vast amount of related literature offered the use of pattern classification methods such as Neural Network, Adaboost, Principal Component Analysis and Support Vector Machine, which adopts metaheuristics to extract important features from a pre-labelled training set. However, most of these individual metaheuristics algorithm are time and memory consuming when used to project the dataset into a lower subspace for subsequent classification. These drawbacks are reduced through a combination of two solution approaches whereby the strengths of two metaheuristics are brought together in a dual level algorithm.

This research presents a dual level metaheuristic algorithm for feature dimensionality reduction using ant colony optimization (ACO) algorithm at Level 1 and genetic algorithm (GA) at Level 2. The dimension of the features in a face dataset will be reduced by using ACO at Level 1 to extract the relevant facial features in the training dataset. The output obtained will be fed into GA at Level 2 to select the discriminant feature subset that becomes the optimized parameter used by support vector machine (SVM) for classification. The developed algorithm suggests that the combination of one or more nature inspired metaheuristic algorithm is crucial for obtaining a high performance optimizer for feature dimensionality reduction to solve the problem of classification.
\end{abstract}

\section{General Terms}

Pattern Recognition.

\section{Keywords}

discriminant feature, metaheuristic algorithm, dual level, dimensionality reduction.

\section{BACKGROUND TO THE STUDY}

Feature dimensionality reduction is the process of reducing the number of features in high dimensional feature space [12]. The problem of dimensionality reduction, encompassing both feature extraction and feature selection, had been the subject of study in a diverse spectrum of fields [10]. The inclusion of irrelevant and redundant features in the dataset can result in poor predictions and high computational overhead. Thus, selecting relevant feature subsets can help reduce the computational cost of feature measurement, speed up learning process and improve model interpretability [4]. Human faces are domain specific features of an image. The feature extraction step extracts the most relevant facial features from the image dataset. These features could be certain face regions, or distances and angles between the parts of the face [15]. Face detection is the process of extracting and locating faces in an image. Face detection has many applications including security applications, human computer interaction, access control, crowd surveillance, to mention but a few. Face detection is a way of detecting the presence of a face in a determined region of the image [7]. The related task of face detection has direct relevance to face recognition because images must be analyzed and faces identified, before they can be recognized. Face recognition involves comparing an image with a database of stored faces in order to identify the individual in that input image. Support Vector Machines (SVMs) have become one of the most popular methods in machine learning in the last twenty years [18]. SVM is a classifier algorithm introduced by Boser and Vapnik (1992). It has high accuracy and flexibility in modelling diverse sources of data such as gene expression, handwritten character recognition, face detection, pedestrian detection and text categorization [11].However, SVM classifier has proven inefficient in its inability to produce accurate classification results in the face of large dataset while it also consumes a lot of computational resources. Hence the need to optimize the classification strength of SVM by ensuring that the resultant feature vectors introduced into the SVM are reduced so as to realize efficient classification [4]. Moreover, the process of choosing appropriate set of features is critical and metaheuristic algorithms offer better and more flexible solution choices. Metaheuristics are nature inspired computational methods that are used to find optimal subset of object from a finite set of objects. They can be employed to obtain a limited yet salient feature set that simplifies pattern representation and builds efficient classifiers. Some of the widely used metaheuristics are genetic algorithm (GA), simulated annealing (SA), particle swam optimization (PSO), ant colony 
optimization algorithm (ACO), tabu search (TS), harmony search (HS), clonal selection algorithm (CSA) and so on.

Nature inspired metaheuristic exhibits synergy. That is, they have the ability to compliment the solution from each other, rather than being competitive. The optimization strategy employed could be that one metaheuristic will search for promising regions in the solution space and the other one is next used in the local search to further prompt the convergence to the global optimum [1]. Two metaheuristics can also be used simultaneously to optimize, fine-tune, and control the parameters of each other, which results in efficient optimization [17]. The combination of two metaheuristic in a dual level fashion will give a greater result in addressing challenging optimization problems such as face detection and recognition as opposed to using standalone metaheuristic for solving optimization problems. ACO is a unique nature inspired computational approach. It is a constructive population based metaheuristic. It exploits an indirect form of memory of previous performance. These features are not found in any other metaheuristic. It employs the use of global search and has high convergence speed but may generate suboptimal solution. GA on the other hand uses local search, converges slowly, but is guaranteed to obtain optimal solution. Hence, the bottom line is to obtain optimal solution by applying ACO to search for local optimality so as to optimize the global final solution.

\section{RELATED WORK}

In general, face recognition techniques can be divided into two groups. They are either feature-based or view/appearance-based. In the former category, human knowledge and facial features such as color, edge, shape, eyes, mouth and some other constraints are used to verify face patterns [19]. In the latter category, 2-D images are directly classified into face groups using pattern recognition algorithms. This approach uses face knowledge implicitly in the system through learning or training face (or nonface) images [14]. [6] developed a GA-ACO algorithm to enhance the performance of GA by incorporating local search and ACO for vehicle routing problem (VRP). The study set up an objective which was to minimize the servicing cost without exceeding the capacity of the vehicles. The simulation results showed satisfactory performance of the GA-ACO algorithm. [5] presented a novel hybrid of ant colony optimization and genetic algorithm as a feature selector, and support vector machine as a classifier integrated efficiently. The work used the combined algorithm to select the optimal feature subsets from features of Persian font images and it was revealed that the number of features becomes nearly half of the original feature number. [2] introduced GA ideas into ACO to present a new binary-coding based ACO. This was found to outperform the typical ACO which has the problem of parameter-space, being replaced with coding space by linking ACO with GA so that the fruits of GA can be applied to ACO directly. Based on the algorithm, it was proved that if the pheromone remainder factor $\rho$ is under the condition of $\rho \geq 1$, the algorithm converges at the optimal, whereas if $0<\rho<1$, it does not. [16] proposed a face recognition system using GA and ACO. The extracted features were saved into memory and GA was used for recognition of unknown face. The work does not induce a classifier for recognition and satisfactory performance was recorded. [13] presented an ACO-based feature selection for face recognition system that uses discrete cosine transform (DCT) to extract features and ACO for face recognition. The work uses nearest neighbour classifier to evaluate the selected feature subset. The result obtained showed superiority of the proposed method. The results obtained from the implementation of the reviewed techniques indicated that better recognition performance can be obtained with nature inspired computational techniques.

\section{THE PROPOSED ALGORITHM}

The ACO-GA procedure for the proposed feature dimensionality reduction is presented in Figure 1.

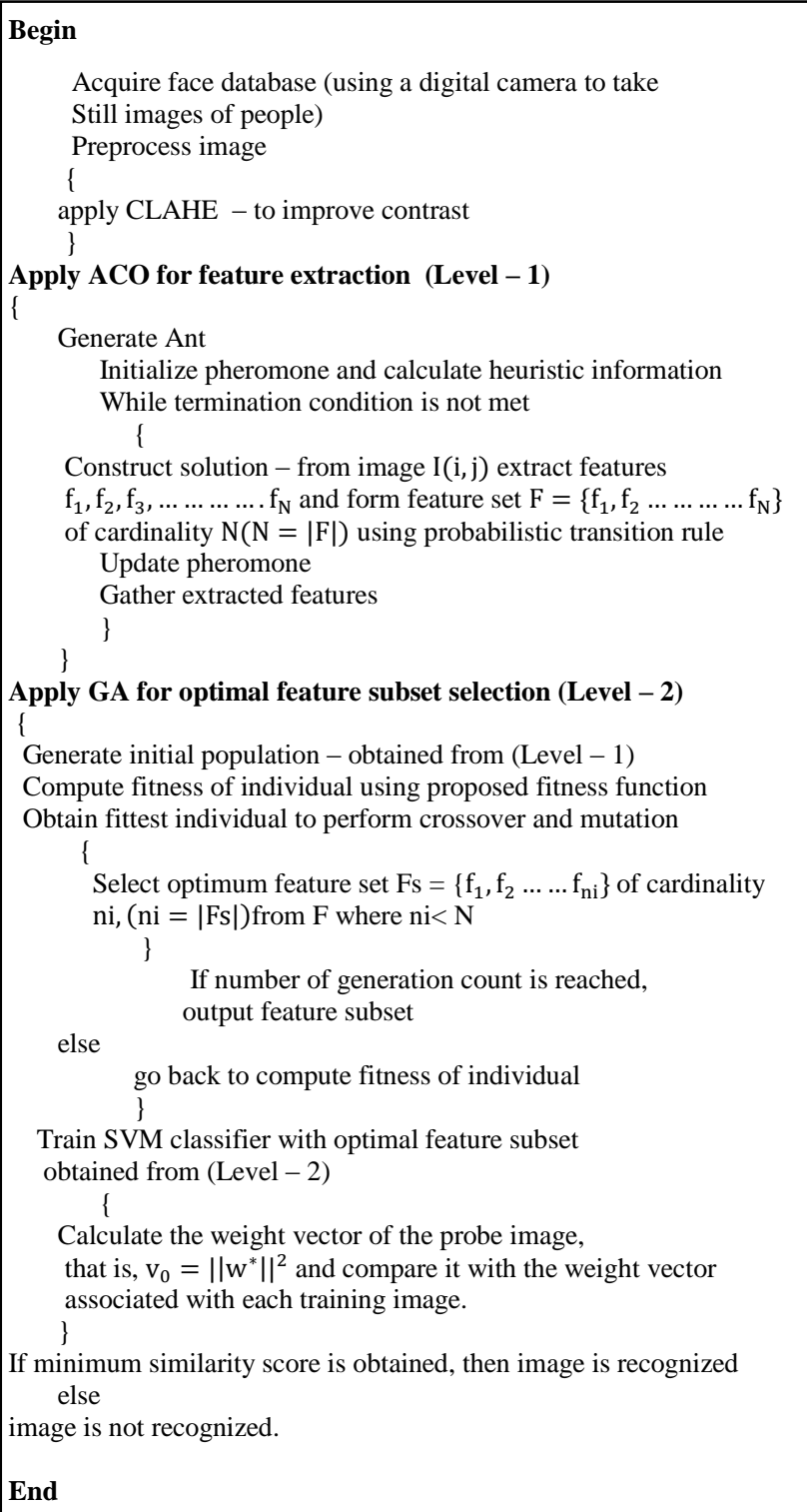

Figure 1: Proposed ACO-GA procedure

\section{PROCESS FLOW OF THE ALGORITHM}

The process flow for the proposed dual level metaheuristic algorithm for feature dimensionality reduction is shown in Figure 2. Details of levels 1 and 2 as proposed are shown in Figure 3 and 4 respectively. 


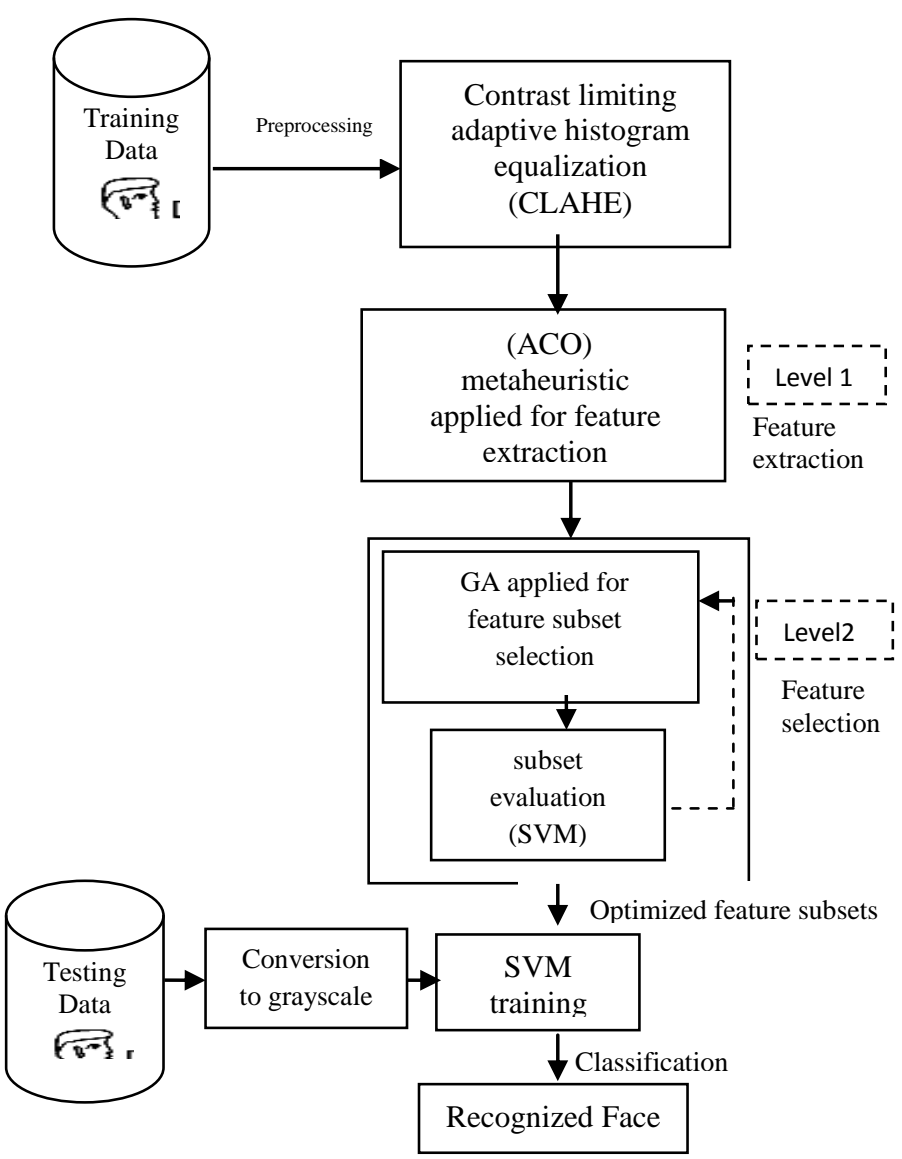

Figure 2: Process flow of the Dual level Algorithm

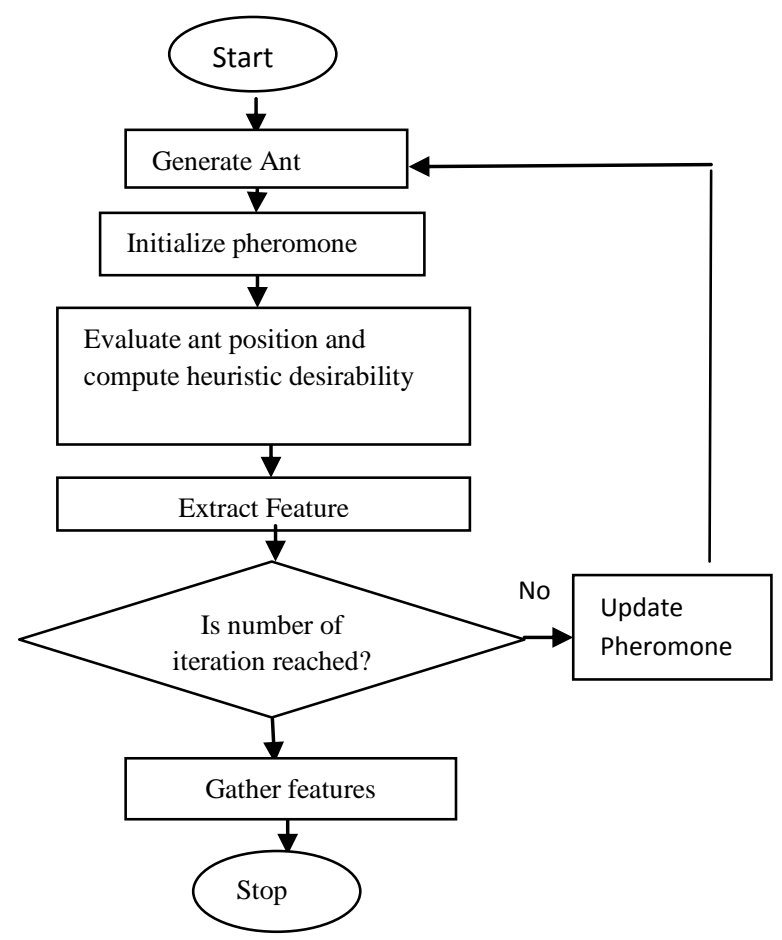

Figure 3: The flowchart for feature extraction using ant colony optimization algorithm as level-1.

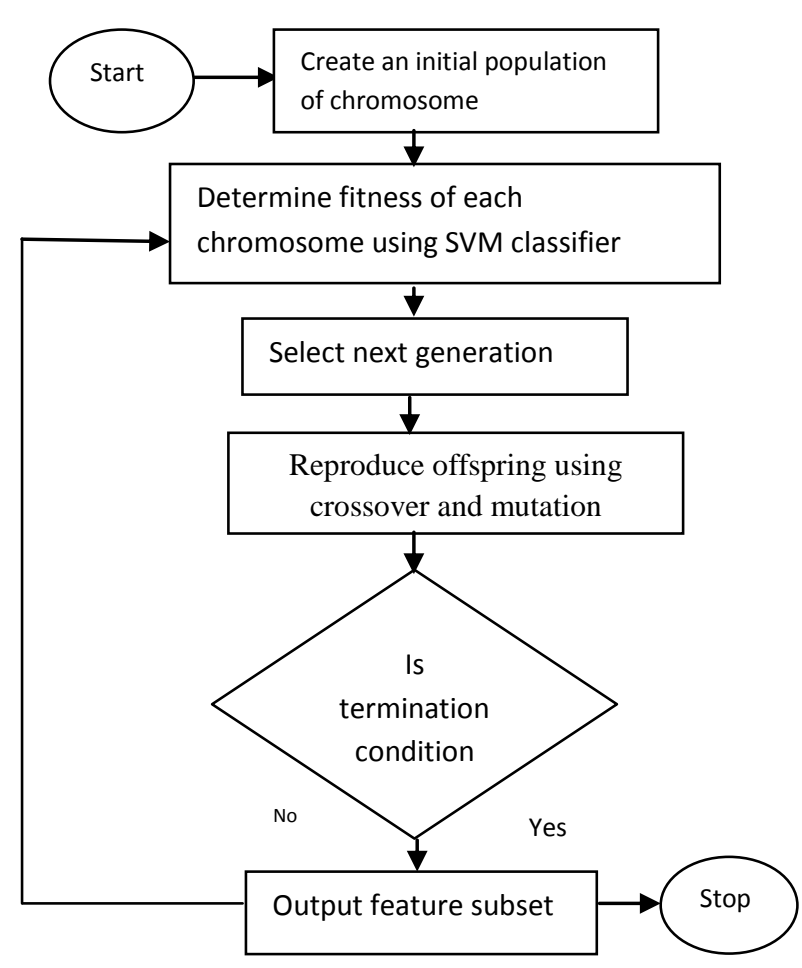

Figure 4: The flowchart for feature selection using genetic algorithm as level-2.

\section{DISCUSSION.}

The algorithm in Figure 1 depicts the complete process from image acquisition, enhancement of the images via CLAHE, feature extraction using ACO, feature selection using GA and classification using SVM.

The dual level metaheuristic algorithm for feature dimensionality reduction begins by applying ACO algorithm on the preprocessed training dataset at Level-1 to extract relevant facial features from the preprocessed face database. At Level-2, GA will receive the reduced features obtained from the feature extraction in Level-1 and these serve as initial population for GA. The most discriminant feature subsets produced by GA will serve as the optimized parameter which SVM will use to detect and recognize human face as shown in Figure 2.

In Figure 3, the feature extraction process begins by generating artificial ants, distributed over the preprocessed image environment to extract feature/pattern concurrently at pixel position. The ants while roaming through the image, guided by pixel to pixel intensity difference, construct solution by extracting high intensity pixel and depositing pheromone. Eventually all the pixels in the route are determined, the shortest path will be chosen and features will be gathered.

In Figure 4, the selection task involves finding out a group of the most effective features out of the features extracted for classification. The optimal feature subset selected will be those that are invariant in a face and are discriminant features i.e. the eyes, nose and mouth. The subset selection approach employed in this research is the wrapper mode which wraps the evaluation of selected subset around a classifier; in this case SVM is used. 


\section{CONCLUSION AND FURTHER WORK}

This research develops a dual level metaheuristic algorithm for feature dimensionality reduction in face detection and recognition. The algorithm has been developed with the intention of combining the strengths of two nature inspired metaheuristic to reduce the dimension of features in a face dataset for efficient classification. The face recognition technology (FERET) data set will be adopted in this research. The training data extracted from FERET will consists of 200 grayscale images of human heads with views ranging from frontal to left and right profiles will be preprocessed to improve contrast using contrast limited adaptive histogram equalization (CLAHE) technique. The next intent is to simulate the algorithm in MATLAB 7.0 toolbox environment and validate the effectiveness on FERET database as well as locally formulated face data set. Performance evaluation on the basis of number of optimum feature subset, classification accuracy and computation time will be carried out for ACOGA-SVM model, ACO-SVM, GA-SVM and SVM for face detection and recognition. The expectation is that the proposed approach to feature dimensionality reduction will contribute an efficient human face detection and recognition.

\section{REFERENCES}

[1] Abraham A., Grosan C. (2008): "Hybridizing a genetic algorithm with an artificial immune system for global optimization". Engineering Evolutionary Intelligent Systems. Springer-Verlag.Vol 38, No. 5, pp. 809 - 814

[2] Bu, Tian-Ming., Yu, Song Nian., Guan, Hui-Wei. (2004): "Binary - Coding - Based Ant Colony Optimization and its Convergence." Vol. 19, No 4, pp. $472-478$.

[3] Delac Kresimir, Grgic Mislav, Grgic Sonja. (2006): "Independent Comparative Study of PCA, ICA and LDA on the FERET Data Set". Wiley Periodicals, Inc. vol. 15.No. 5, pp.252-260.

[4] Fagbola, Temitayo., Olabiyisi, Stephen, Adigun Abimbola. (2012): "Hybrid GA-SVM for Efficient Feature Selection in E-mail Classification". Computer Engineering and Intelligent Systems.Vol.3 No. 3. pp. 1728.

[5] Imani Maryam Bahojb, Pourhabibi Tahereh, Keyvanpour Mohammad Reza, and Azmi Reza. (2012): “A New Feature Selection Method Based on Ant Colony and Genetic Algorithm on Persian Font Recognition". International Journal of Machine Learning and Computing. Vol 2 No. 2.Pp 278-282

[6] Li, Na., Wang, Shoubi., Li Yulan. (2011): "A hybrid Approach of Genetic Algorithm and Ant Colony Optimization for Vehicle Routing Problem". Journal of Computational Information Systems Vol. 7 No. 13.pp 4939 - 4946.

[7] Mall Anjana, Ghosh Shusmita. (2012): "Neural Network training Based Face Detection and Recognition".
International Journal of Computer Science and Management Research. Vol. 1 No. 2.pp.103-109

[8] Osuna,E. Freund, R. and Girosi, F. (1997): "Training Support Vector Machines: An Application to Face Detection," in Proc. IEEE Conf. Computer Vision and Pattern Recognition, pp. 130-136

[9] Platt, C. John. (1998): “ Sequential Minimal Optimization. A fast algorithm for Training Support Vector Machines". Technical Report MSR-TR-98-14.

[10] Raymer, M.L. Punch, W.F., Goodman, E.D., Kuhn, L.A., Jain, A.K. (2000): "Dimensionality Reduction using Genetic Algorithms". IEEE Transactions on Evolutionary Computation, Vol. 4 No. 2. pp. 164-171.

[11] Rifkin, R., Klantan, N. (2004): "In defense of one vs-all classification. Journal of Machine Learning Research, 5:101-141

[12] Guyon, I., Elisseeff, A. (2008): " Special issue on variable and feature selection". Journal of Machine Learning Research. Vol. 3. pp. 1157-1182.

[13] Hamidreza Rashidy, Karim Kanan1Faez1 and Mehdi Hosseinzadeh. (2007): Face Recognition System Using Ant Colony Optimization-Based Selected Features. Proceedings of the 2007 IEEE Symposium on Computational Intelligence in Security and Defense Applications. Pp. 57-52.

[14] Hjelmas, E. and Low, B. K. (2001): "Face detection: A survey". Computer Vision and Image Understanding, vol. 83, pp. 236-274.

[15] Sawalha Rana, Doushlyad Abu. (2012): "Face Recognition Using Harmony Search- Based Selected Features". International Journal of Hybrid Information Technology. Vol. 5. No 2. pp. 1-16

[16] Venkatesan M.E. Srinivasa R M. (2010): Face Detection by Hybrid Genetic and Ant Colony Optimization Algorithm. International Journal of Computer Applications.Vol.9.No.4. pp. 8-13.

[17] Wang, Xiaolei. (2009): "Hybrid Nature-Inspired Computation Methods for Optimization". Doctoral Dissertation. Helsinki University of Technology, Faculty of Electronics, Communications and Automation. Department of Electrical Engineering. Pp. 5-40. Unpublished.

[18] Wu, Xindong., Kumar, Vipin., Quinlan, J. Ross., Ghosh, Joydeep., Yang, Qiang., Motoda, Hiroshi., McLachlan, J. Geoffrey., Ng, Angus., Liu, Bing., Yu, S. Philip., Zhou, Zhi-Hua., Steinbach, Michael., Hand, J. David., Steinberg, Dan. (2008): "Top 10 Algorithms in Data Mining". Survey Paper. Knowledge Information System. Vol. 14. pp. 1-37.

[19] Yang, M.-H., Kriegman,D. and N. Ahuja. (2002): "Detecting Faces in Images: A Survey," IEEE Trans. Pattern Analysis and Machine Intelligence, vol. 24, No. 1, pp. 34-58. 